\title{
Development of a high-density linkage map and mapping of the three-pistil gene (Pis1) in wheat using GBS markers
}

\author{
Zaijun Yang ${ }^{*}$, Zhenyong Chen, Zhengsong Peng, Yan Yu, Mingli Liao and Shuhong Wei
}

\begin{abstract}
Background: The wheat mutant line three-pistil (TP) exhibits three pistils per floret. As TP normally has two or three seeds in each of the florets on the same spike, there is the possibility of increasing the number of grains per spike. Therefore, TP is a highly valuable mutant for breeding and for the study of floral development in wheat. To map the three-pistil gene (Pis1), genotyping-by-sequencing single-nucleotide polymorphism (GBS-SNP) data from an $\mathrm{F}_{2}$ mapping population (CM28 $\times$ CM28TP) was used to construct a genetic map that is of significant value.
\end{abstract}

Results: In the present study, a high-density genetic map of wheat containing 2917 GBS-SNP markers was constructed. Twenty-one linkage groups were resolved, with a total length of $2371.40 \mathrm{cM}$. The individual chromosomes range from $2.64 \mathrm{cM}$ to $454.55 \mathrm{cM}$ with an average marker density of $0.81 \mathrm{cM}$. The Pis1 gene was mapped using this high-resolution map, and two flanking SNP markers tightly linked to the gene, M70 and M71, were identified. The Pis 1 is $3.00 \mathrm{cM}$ from M70 and $1.10 \mathrm{cM}$ from M71. In bread wheat genome, M70 and M71 were found to delimit a physical distance of 3. $40 \mathrm{Mb}$, which encompasses 127 protein-coding genes. To validate the GBS-generated genotypic data and to eliminate missing marker data in the Pis1 region, five Kompetitive Allele-Specific PCR (KASP) assays were designed from corresponding GBS sequences, which harbor SNPs that surround Pis1. Three KASP-SNP markers, KM70, KM71, and KM75, were remapped to the Pis 1 gene region.

Conclusions: This work not only lays the foundation for the map-based cloning of Pis 1 but can also serve as a valuable tool for studying marker-trait association of important traits and marker-assisted breeding in wheat.

Keywords: Wheat, Genotyping-by-sequencing (GBS), Three-pistil mutation, Kompetitive allele-specific PCR (KASP)

\section{Background}

Wheat (Triticum aestivum L.), which is considered to be the second-most consumed crop, is cultivated widely around the world. However, in recent years, levels of wheat production have not satisfied the global demand, triggering price instability and hunger riots. Accordingly, a major goal of wheat production is to increase yield. One effective method of improving wheat yield is to increase the number of grains per spike [1,2], which can be achieved through a wide-range of genetic variations in the morphological framework of wheat, including superfluous spikelets and multiple spikelets [3] as well as multiple rows of spikes [4]. Described by

\footnotetext{
*Correspondence: yangzaijun1@126.com

Key Laboratory of Southwest China Wildlife Resources Conservation (Ministry of Education), College of Life Science, China West Normal University, Nanchong, Sichuan 637009, China
}

Peng, three-pistil (TP) mutant wheat has normal spike morphology but exhibits three pistils per floret [5]. As a result, three seeds can be cultivated per floret, increasing the number of seeds per spike. Previous studies have shown that the three-pistil trait is controlled by the Pis1 gene, a dominant gene located on chromosome arm 2DL $[5,6]$ between simple sequence repeat (SSR) markers Xgwm539 and Xgwm349 [7]. However, at $17.6 \mathrm{cM}$ and $19.5 \mathrm{cM}$ from Xgwm539 and Xgwm349, respectively, Pis1 is too distant to meet the requirements for fine-mapping [7]. In recent years, our group has explored genes contributing to the three-pistil trait in wheat. Although a large number of relevant genes have been cloned [8-11], the Pis1 gene has not. Map-based cloning is the best approach for the cloning of Pis1, the first step of which is to find markers closely linked to Pis1 gene. Nonetheless, common wheat $(2 n=6 x=42)$ has a large genome $(16 \mathrm{~Gb})$, with 
highly repetitive sequences; thus, fine-mapping of functional genes is challenging.

Recently, scientists have been able to determine allelic variations corresponding to complicated traits with the help of advances in next-generation sequencing (NGS) technology [12]. Several methods that combine marker discovery and genotyping have been developed, such as reducedrepresentation sequencing, restriction-site-associated DNA sequencing (RAD-seq), and low-coverage genotyping, which includes multiplexed shotgun sequencing (MSG) and genotyping-by-sequencing (GBS). To some extent, these approaches are similar in technique. By generating the same amount of data per sample with a 96-plex library, GBS is beneficial for improving outcomes and reducing the cost per sample, making this genotyping platform quite appealing [13]. The two- or three-enzyme GBS approach can be applied to genotype species with large and complex genomes, such as barley, or species with polyploid genomes, such as common wheat [13]. Developing high-density GBS markers in hexaploid wheat may promote progress in the identification of the physical locations of genes of interest.

According to latest research, we constructed a highdensity linkage map of wheat based on an $\mathrm{F}_{2}$ population of 200 individuals derived from cross of Chuanmai 28 (CM28) with its near-isogenic line CM28TP using SNP markers obtained via GBS technology. The Pis1 gene was then mapped using this high-density linkage map. This work not only lays the foundation for map-based cloning of the Pis1 gene but can also serve as a valuable tool for the identification and genetic dissection of many other complex and important wheat traits in the future.

\section{Results}

\section{Sequencing the parental lines and the $\mathrm{F}_{2}$ population}

The parental lines CM28TP and CM28 were sequenced using GBS at efficient sequencing depths of approximately 23.3-fold and 19.0-fold, respectively. With regard to CM28TP and CM28, 29,464,252 and 21,332,415 reads, respectively, were mapped to the sequence of the bread wheat genome (IWGSC1+popseq.31.pep, ftp://ftp.ensemblgenomes. org/pub/plants/release-31/fasta/triticum_aestivum/pep/). A total of 727,097 and 679,440 SNPs were identified in CM28TP and CM28, respectively. For the $\mathrm{F}_{2}$ population, the efficiency of enzyme digestion was quite high, at $94 \%$. A total of $1,862,290,768$ reads were produced, with an average of 9,311,454 reads per individual; this is equivalent to approximately 0.23 -fold coverage of the bread wheat genome. The average GC content of the sequences is $40.5 \%$, with a Q20 score of $93.9 \%$. Given that the two parents are homozygous inbred lines with $a a$ and $b b$ genotypes, only the genotype $a a \times b b$, consisting of 21,584 markers, was used for further analysis. Among the 21,584 markers, the low-coverage sequences of the $F_{2}$ population (coverage less than $75 \%$ ) were filtered, leaving 16,475 markers.
Markers with significant distortion $(p<0.001)$ were filtered, and 3655 markers were retained in total with the purpose of determining bin markers.

\section{Genetic linkage map with bin markers}

A total of 2917 SNPs (1987 bin markers) were mapped to 21 linkage maps; 738 SNPs were unanchored to any chromosome (Fig. 1, Additional file 1: Figure S1). These genetic maps spanned a total length of $2371.40 \mathrm{cM}$, with individual chromosomes ranging from $2.64 \mathrm{cM}(6 \mathrm{D})$ to $454.55 \mathrm{cM}$ (3B) (Table 1). The average distance is 0.81 . The number of bin markers in the different chromosomes range from 2 (4D and 6D) to 192 (3B) and are not evenly distributed on each chromosome. A total of 846 bin markers were mapped to the A genome, covering a distance of $690.27 \mathrm{cM}$. For the B genome, 905 bin markers were mapped, covering a distance of $1255.69 \mathrm{cM}$. However, only 236 bin markers were mapped to the D genome, covering a distance of $425.44 \mathrm{cM}$ (Table 1). A total of 87 gaps from $5 \mathrm{cM}$ to $45 \mathrm{cM}$ were observed, and the largest gap, $44.39 \mathrm{cM}$, was found on chromosome 4D (Table 1).

To verify this map, we used the sequences of 1987 bin markers in a BLASTn query to search the bread wheat genome (Chinese Spring) (Additional file 1: Table S1). All the markers can hit to the bread wheat genome sequence, and all the sequence of markers can be mapped to the same chromosome in the bread wheat genome sequence.

\section{Mapping of the Pis1 gene}

To map Pis1, we recorded the number of pistils per floret for the $200 \mathrm{~F}_{2}$ individuals during the flowering period. The pistils were divided into two groups: three pistils or one pistil per floret. The ratio of three pistils to one pistil fit to $3: 1\left(x^{2}=0.96, p>0.05\right)$, indicating that a single dominant gene controls the three-pistil trait. A high logarithm of the odds (LOD) score of 60.4 was detected for chromosome 2D (Fig. 2a). The genetic linkage map of chromosome 2D was constructed using pistil phenotypic and SNP data. Two markers were found to be tightly linked to the Pis1 gene, namely, M70 and $M 71$, with genetic distances of $3 \mathrm{cM}$ and $1.1 \mathrm{cM}$ from Pis1, respectively. The physical distance of $M 70$ and $M 71$ is $3.4 \mathrm{Mb}$ in the sequence of the bread wheat genome (Chinese Spring) (Fig. 2b).

To verify the GBS-generated genotypic data and to eliminate missing marker data in the Pis1 region, five KASP markers were designed from corresponding GBS sequences harboring SNPs surrounding Pis1 (Table 2). To avoid confusion with GBS-SNP markers, we tentatively designated the KASP-SNP markers as KM69, KM70, KM71, KM75, and KM76, which correspond with M69, M70, M71, M75, and M70 in GBS-SNP markers, respectively. Four KASP-SNP markers were well amplified 


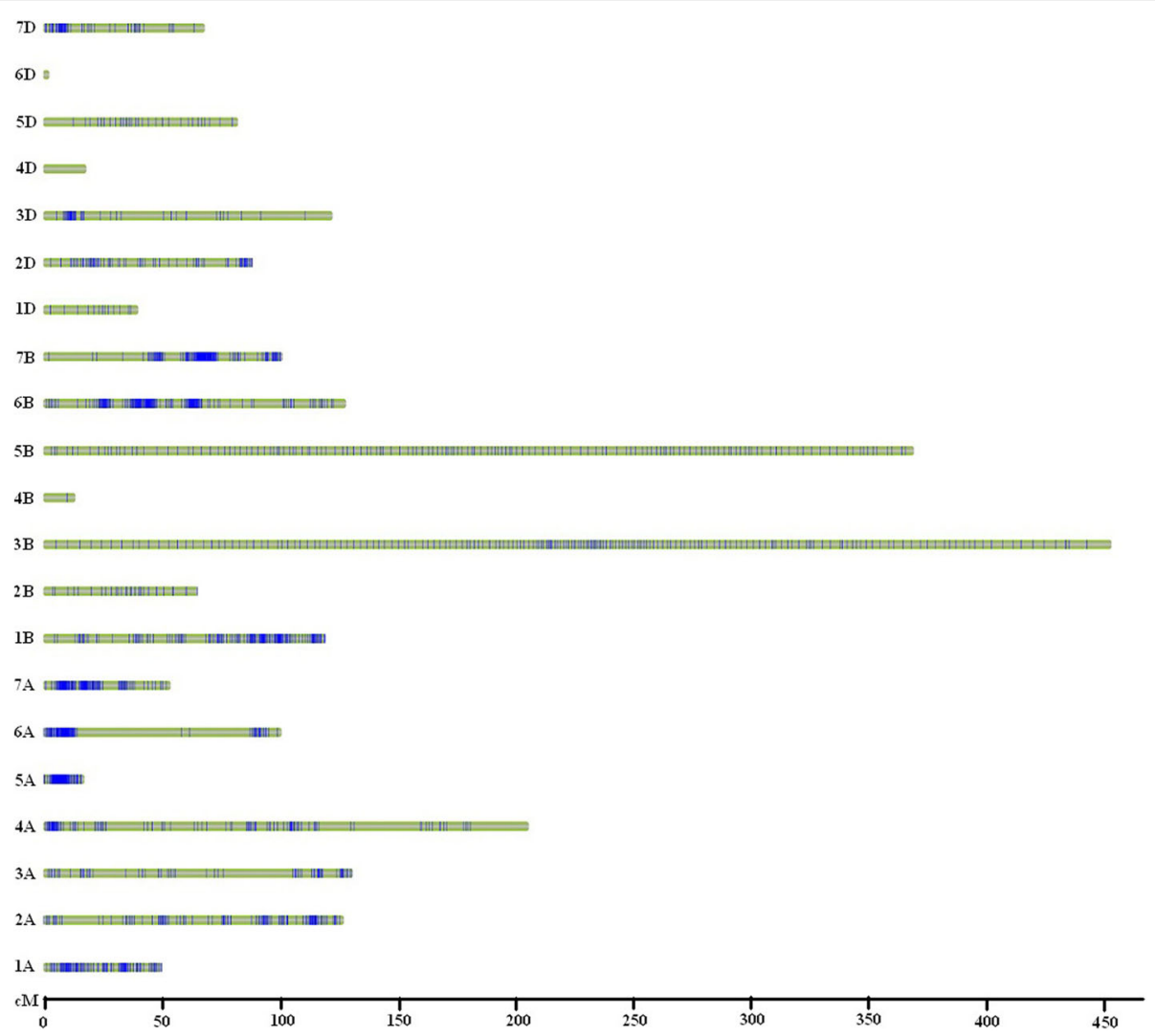

Fig. 1 Distribution of the GBS markers on 21 linkage groups. The genetic distances (cM) are shown on the $x$-axis

and polymorphic between the parents and in the $\mathrm{F}_{2}$ population. Three markers, $K M 70, K M 71$, and $K M 75$, were remapped to the Pis1 region. The KASP-SNP KM69 is not linked to Pis1. A comparison of the GBS-SNP and KASPSNP data revealed identical genotypes in the $\mathrm{F}_{2}$ population for KM70, KM71, and KM75. In contrast, the KASP-SNP marker KM69 did not match the GBS-SNP data because of calling errors for GBS-SNP or KASP-SNP data in $114 \mathrm{~F}_{2}$ individuals.

\section{Candidate gene prediction and functional annotation}

According to the wheat gene annotation database, Triticum_aestivum.IWGSC1+popseq.31.pep (ftp://ftp. ensemblgenomes.org/pub/plants/release-31/fasta/triticum aestivum/pep/), the physical intervals of $M 70$ and M71 encompass 127 protein-coding genes. Currently, 91 protein functions have been annotated in this region, among which 83 have Gene Ontology (GO) annotations (Additional file 1: Table S2). The results of GO analysis indicated that the number of genes in the different classifications varies: 107 genes were identified in the cellular component category, 552 genes in the molecular function category, and 326 genes in the biological process category. Several genes have multiple functions, and these were categorized into more than one function. In the cellular components category, 17 genes are related to the cell and 17 to cellular ontology. In the molecular functions category, 55 genes are associated with binding. In the biological processes category, 47 genes are relevant to metabolic processes (Fig. 3). Among the candidate genes within a 1.5-LOD decrease on either side of the peak bin that was delimited as the Pis1 interval (the physical distance ranges from 139,248,092 bp to $139,416,092 \mathrm{bp}), 10$ genes were identified with the closest proximity to Pis1. Of these 10 genes, annotation information is available for 6 (Table 3). Among them, 3 are associated with molecular functions, 2 with cellular components, and only one with biological processes.

\section{Discussion}

Although TP, a common wheat mutant, possesses normal spike morphology, it exhibits three pistils per floret; thus, TP has the potential for increased wheat production [6]. It has been demonstrated by genetic analysis that the TP trait is under the control of a single dominant nuclear gene, Pis1, which lies between SSR markers Xgwm539 and Xgwm349 on chromosome arm 2DL [7]. However, Pis1 is too genetically distant from Xgwm539 and Xgwm349 to meet the requirements for fine-mapping. Pis1 marker development has presented considerable 
Table 1 Marker information for the high-density genetic map

\begin{tabular}{|c|c|c|c|c|c|c|}
\hline Chr. & No. SNP & No. Bin marker & Genetic distance (cM) & Average distance (cM) & Max. gap (cM) & $<5$ cM gap \\
\hline $1 \mathrm{~A}$ & 119 & 119 & 50.43 & 0.42 & 2.51 & 119 \\
\hline $2 \mathrm{~A}$ & 132 & 132 & 128.02 & 2.37 & 10.44 & 128 \\
\hline $3 \mathrm{~A}$ & 75 & 75 & 131.82 & 4.59 & 10.13 & 68 \\
\hline $4 \mathrm{~A}$ & 121 & 121 & 206.66 & 2.41 & 5.70 & 112 \\
\hline $5 \mathrm{~A}$ & 242 & 135 & 17.60 & 0.71 & 12.44 & 134 \\
\hline $6 \mathrm{~A}$ & 125 & 125 & 101.49 & 0.59 & 18.61 & 122 \\
\hline $7 \mathrm{~A}$ & 239 & 139 & 54.25 & 2.70 & 5.89 & 137 \\
\hline $1 B$ & 286 & 165 & 120.18 & 1.16 & 9.20 & 159 \\
\hline $2 B$ & 38 & 38 & 65.78 & 2.62 & 18.75 & 34 \\
\hline $3 B$ & 388 & 192 & 454.55 & 9.18 & 18.36 & 186 \\
\hline $4 B$ & 3 & 3 & 13.77 & 2.59 & 12.81 & 1 \\
\hline $5 B$ & 267 & 154 & 370.51 & 0.97 & 15.63 & 151 \\
\hline $6 B$ & 345 & 181 & 128.90 & 1.32 & 2.64 & 175 \\
\hline 7B & 301 & 172 & 101.99 & 1.13 & 10.69 & 165 \\
\hline $1 D$ & 15 & 15 & 40.50 & 1.76 & 29.53 & 12 \\
\hline $2 \mathrm{D}$ & 77 & 77 & 89.07 & 1.71 & 28.28 & 75 \\
\hline $3 \mathrm{D}$ & 47 & 47 & 123.16 & 0.13 & 1.37 & 38 \\
\hline $4 \mathrm{D}$ & 2 & 2 & 18.36 & 0.81 & 44.39 & 0 \\
\hline $5 \mathrm{D}$ & 32 & 32 & 82.96 & 0.39 & 6.64 & 27 \\
\hline $6 \mathrm{D}$ & 2 & 2 & 2.64 & 0.73 & 8.65 & 1 \\
\hline $7 D$ & 61 & 61 & 68.76 & 1.73 & 5.57 & 56 \\
\hline total & 2917 & 1987 & 2371.40 & 0.81 & 44.39 & 1900 \\
\hline A & 1053 & 846 & 690.27 & 0.66 & 18.61 & 820 \\
\hline B & 1628 & 905 & 1255.69 & 0.77 & 18.75 & 871 \\
\hline D & 236 & 236 & 425.44 & 1.80 & 44.39 & 209 \\
\hline
\end{tabular}

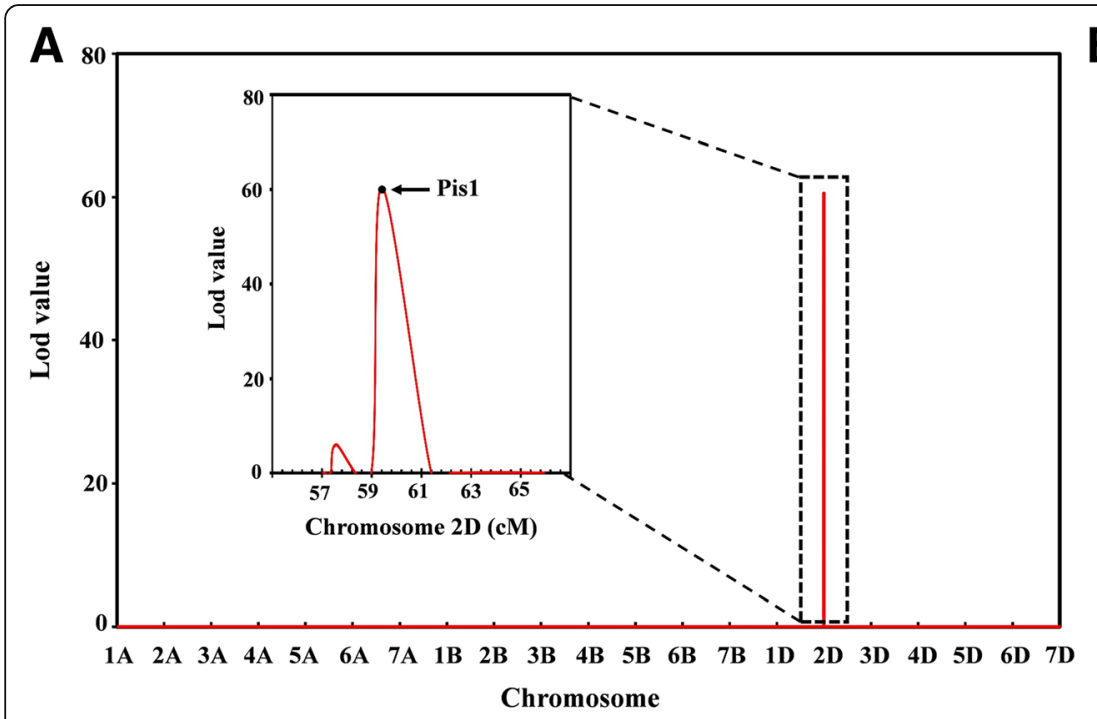

B

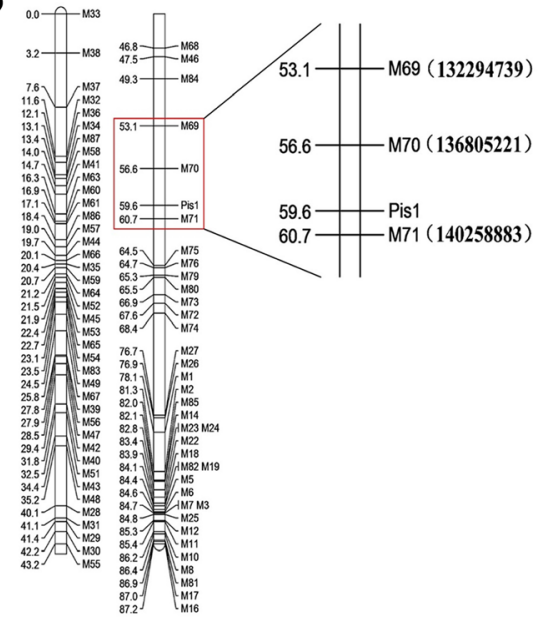

Chromosome 2D

Fig. 2 Detailed mapping of the Pis1 gene on chromosome 2D. a The result of the QTL mapping of the three-pistil trait in wheat. Mapping QTL for plant height was conducted in the $F_{2}$ population of CM28 and CM28TP in MapQTL6.0. In the chart, the red curve indicated LOD value at any genetic position. $\mathbf{b}$ The genetic linkage map of the Pis 1 gene. Genetic distances are indicated on the left side of linkage group in centiMorgans (cM), and the marker names are shown on the right side. The physical distances are shown in the brackets 
Table 2 Primer sequences for the KASP assays of the GBS markers linked to the Pis 1 gene

\begin{tabular}{llll}
\hline Markers & Allele-specific 1 (FAM) & Allele-specific 2 (HEX) & Common \\
\hline KM69 & AACAAAAGCGGGTCCTCTCCG & GAACAAAAGCGGGTCCTCTCCA & CGTGCTGCTTCCTTCCAAGCCAT \\
KM70 & ATCGCCAGATGCCACGCACAA & CGCCAGATGCCACGCACAG & AGGGACGTGATCAAATTTCTTGACGATT \\
KM71 & TITGGACATTATTGGGCTTATTATACAC & GTITGGACATTATTGGGCTTTATTATACAA & CTGGGTTAATAGGTTAGTCCCAAAAGTAA \\
KM75 & GGTGCTCGCCCTAAACAATCACA & GTGCTCGCCCTAAACAATCACC & CAGTCCACGTGTCTTTCTGTAAAACATT \\
\hline
\end{tabular}

challenges due to the relatively low level of DNA polymorphisms detected on chromosome 2D between wheat genotypes. Only 61 SSR markers are available for 2D, which is reflected in the low marker density of chromosome 2D linkage maps [14]. After exhaustively testing available SSR markers, none were sufficiently close to Pis1 to ensure its reliable detection. Therefore, GBS was chosen as a new approach for mapping of the Pis1 gene.

GBS is a recently developed, NGS-based genotyping approach that is cost-effective and highly accurate for high-density marker development [15]. The use of GBS markers is advantageous in several aspects. For instance, GBS has a general sample preparation method, a highly robust genome complexity reduction strategy to facilitate de novo marker discovery across entire genomes, and a uniform bioinformatics workflow strategy to achieve genotyping goals tailored to individual species that is independent of a reference sequence [12]. The most extraordinary attribute of GBS is the ability to genotype any population structure regardless of the availability of parental data and the ability to co-dominantly score SNP markers segregating in populations [12]. We utilized this novel genotyping method to map Pis1 in an $\mathrm{F}_{2}$ population. The development and application of GBS markers in an $F_{2}$ population are examples of the successful development of high-density markers in wheat, for which there is no sequenced genome available, and the determination of the physical location of a major gene without sequencing physical contigs or the complete wheat genome.

In this work, the benefits of the GBS protocol are exemplified by successful genotyping of an $F_{2}$ population and generating abundant parental SNP information with a low cost and effort. The $\mathrm{F}_{2}$ population was derived from crossing CM28 with its near-isogenic line CM28TP. CM28 and CM28TP have similar phenotypes and genetic backgrounds, except for the three-pistil phenotype [8]. Polymorphisms were minimal in this $F_{2}$ population; however, we believe that sequencing of this population could be used to identify markers closely linked to Pis1. In total, 727,097 and 679,440 SNPs were identified in CM28TP and CM28, respectively, and 2917 selected polymorphic markers were eventually identified for the construction of a high-density linkage map. The 2917

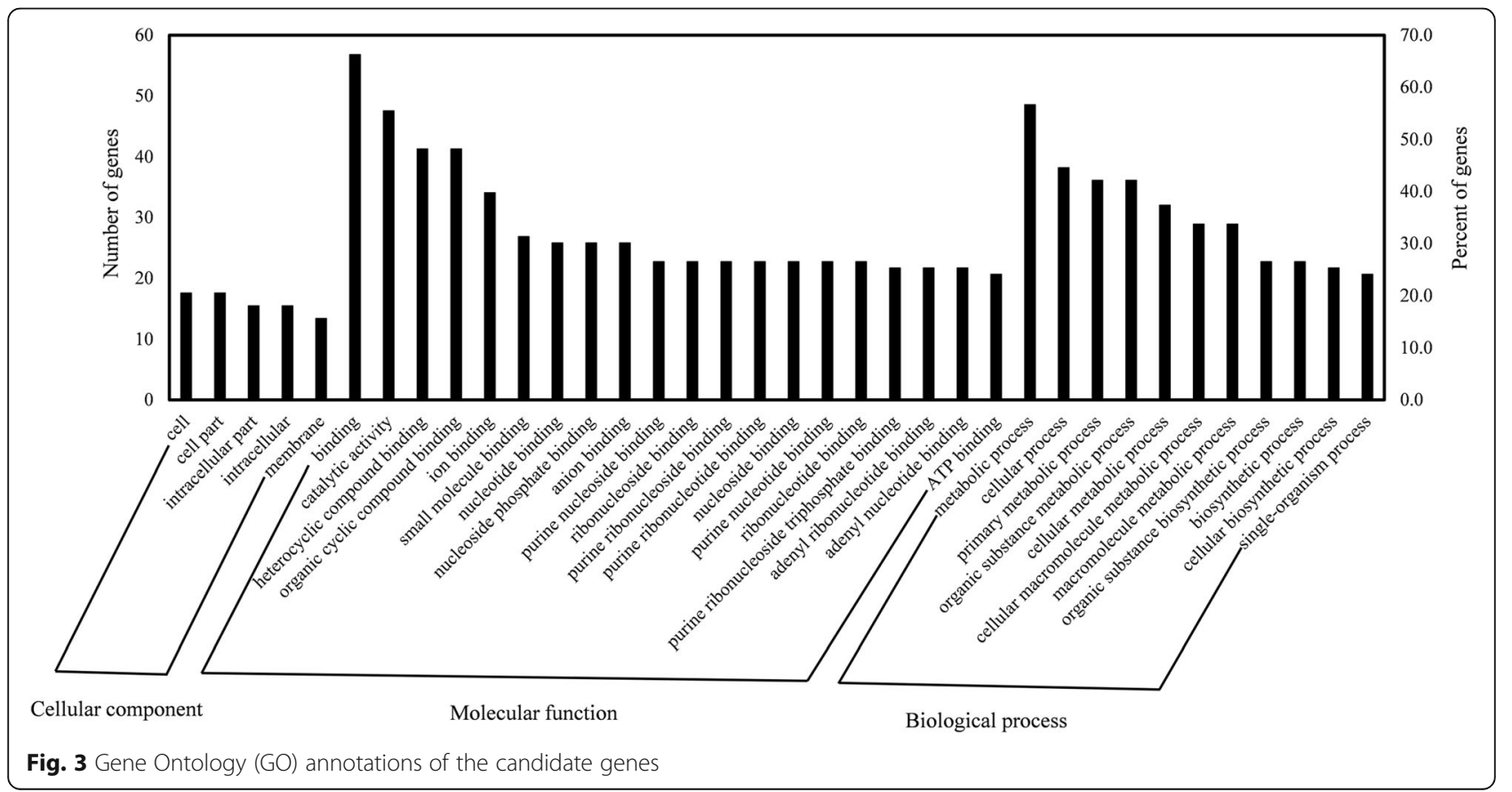


Table 3 The positions of the candidate genes nearest to Pis 1

\begin{tabular}{|c|c|c|c|c|c|c|}
\hline No. & Start position & End position & $\begin{array}{l}\text { BLAST matching } \\
\text { accession no. }\end{array}$ & Annotation & Sequence identity (\%) & E value \\
\hline 1 & $139,244,852$ & $139,245,784$ & EMT28401 & N/A & & \\
\hline 2 & $139,342,698$ & $139,344,779$ & ACT22500 & plastid glutamine synthetase 2 [Triticum aestivum] & $98 \%$ & $9 e-66$ \\
\hline 3 & $139,348,585$ & $139,351,702$ & EMS55336 & DNA topoisomerase 1 [Triticum urartu] & $80 \%$ & 0 \\
\hline 4 & $139,364,799$ & $139,366,357$ & EMS51822 & Transmembrane 9 superfamily member 4 [Triticum urartu] & $97 \%$ & 0 \\
\hline 5 & $139,369,519$ & $139,370,195$ & EMT08392 & Tropinone reductase-like protein [Aegilops tauschii] & $88 \%$ & $4 e-53$ \\
\hline 6 & $139,380,317$ & $139,381,181$ & EMT03631 & N/A & & \\
\hline 7 & $139,385,118$ & $139,386,329$ & EMS51820 & N/A & & \\
\hline 8 & $139,393,899$ & $139,394,513$ & EMT03801 & Transmembrane 9 superfamily member 2 [Aegilops tauschii] & $98 \%$ & $1 e-93$ \\
\hline 9 & $139,406,363$ & $139,408,447$ & EMT09611 & U3 small nucleolar ribonucleoprotein IMP3 [Aegilops tauschii] & $99 \%$ & $2 e-130$ \\
\hline 10 & $139,416,283$ & $139,418,780$ & NP_001150358 & N/A & & \\
\hline
\end{tabular}

SNPs cover all 21 linkage groups, spanning a total length of $2371.40 \mathrm{cM}$. To the best of our knowledge, this genetic map represents the densest map in wheat thus far. The mean distance between two neighboring markers, $0.81 \mathrm{cM}$, is the least compared with the previously reported mean distances of $0.88 \mathrm{cM}$ and $0.86 \mathrm{cM}$ $[16,17]$. However, D genome chromosomes were less well represented in this study. In particular, only two markers were found for $4 \mathrm{D}$ and $6 \mathrm{D}$. This poor representation possibly resulted from low polymorphisms of the D genome $[18,19]$.

The development of closely linked markers and finemapping is an essential initial step in the map-based cloning of Pis1. In the present study, we mapped Pis1 using a genetic linkage map that we had constructed. Pis1 is located in a $4.1 \mathrm{cM}$ region and is flanked by $M 70$ and M71. Sequencing-based genotyping technology may result in a large amount of missing data, and even GBS cannot avoid this shortcoming. Therefore, it is suggested that the method of imputation be applied to predict genotypes with missing data $[12,13,20,21]$. Utilizing high-quality SNPs with $<20 \%$ missing data without imputation is an alternative for improving the data quality [22], though this approach will likely lead to some essential SNPs being overlooked. During this research, we utilized GBS-SNP with $<25 \%$ missing data to construct a map for delineating Pis1. To verify the GBS-SNP data, we then converted GBS-SNPs closely linked to Pis1 to KASP-SNPs. With this approach, the negative effect caused by missing data and corrected sequencing error can be minimized, therefore improving the accuracy of the results for Pis1 mapping. Among the five designed KASP assays, were successful in the $\mathrm{F}_{2}$ population, three corresponded to GBS-SNP calls (markers KM70, KM71, and KM75), and one had SNP call errors. These errors are likely to occur in sequencing and during the SNPcalling pipeline. Therefore, we can conclude that the Pis1 gene location is accurate. M70 and M71 have a physical distance of 3.4 Mb in the sequence of the bread wheat genome and encompass 127 protein-coding genes. Although the relationship between the 127 identified genes and the three-pistil trait requires further research, our study on mapping of the Pis1 gene lays the foundation for the map-based cloning of Pis1.

\section{Conclusions}

In the present study, a high-density linkage map of wheat was constructed using GBS-SNP data from an $F_{2}$ mapping population $(\mathrm{CM} 28 \times \mathrm{CM} 28 \mathrm{TP})$. The map spans a total length of $2371.40 \mathrm{cM}$, and individual chromosomes range from $2.64 \mathrm{cM}$ to $454.55 \mathrm{cM}$. The average distance is $0.81 \mathrm{cM}$. Using this map, the Pis1 gene was mapped, and two markers tightly linked to the Pis1 gene, M70 and M71, were identified. The Pis1 gene is located between SNP markers $M 70$ and $M 71$ and is $3 \mathrm{cM}$ from M70 and $1.1 \mathrm{cM}$ from $M 71$. M70 and M71 have a physical distance of $3.4 \mathrm{Mb}$ in the draft sequence of the bread wheat genome, which encompasses 127 proteincoding genes. To validate the GBS-generated genotypic data and to eliminate missing marker data in the Pis1 region, five KASP markers were designed from corresponding GBS sequences harboring SNPs surrounding Pis1. Three KASP-SNP markers, KM70, KM71, and KM75, were remapped to the Pis1 gene region. This work not only lays the foundation for the map-based cloning of Pis1 but can also serve as a valuable tool for studying marker-trait association of important traits and marker-assisted breeding in wheat.

\section{Methods}

Plant materials and DNA isolation

This study utilized a near-isogenic line, CM28TP (carrying the Pis1 gene), and its recurrent parent Chuanmai 28 (CM28). CM28TP has a similar phenotypic to CM28, except for the three-pistil trait [8]. An $F_{2}$ population of 200 individuals derived from the cross between CM28 and 
CM28TP was used to construct the map. All plants were maintained at the China West Normal University in Nanchong, China. Fresh leaves from the parents and $F_{2}$ individuals were collected for DNA isolation. Samples were lyophilized and stored at $-80{ }^{\circ} \mathrm{C}$ until use. Total genomic DNA was isolated with Plant Genomics DNA Kit (TIANGEN Biotech) in accordance with the manufacturer's recommendations. The quality and concentration of the genomic DNA were assessed by agarose gel electrophoresis and using an ND-2000C spectrophotometer (NanoDrop).

\section{Genotyping-by-sequencing (GBS) approach}

DNA from the $200 \mathrm{~F}_{2}$ plants and two parental plants was subjected to GBS according to Elshire et al. [23]. Specifically, $200 \mathrm{ng}$ of DNA from each sample was digested with a combination of MseI, HaeIII, and EcoRI restriction enzymes, and barcoded forward adapters and common reverse adapters were ligated to the digested fragments. A total of 202 samples were included in one 202-plex library. The PCR reactions were performed using Illumina primers with sequences complimentary to the adapters used during the library preparation. The PCR products were sent to Beijing Novogene Bioinformatics Technology Co., Ltd. for next-generation sequencing (NGS) using an Illumina Hi-seq 2000 platform. One library was double-loaded onto two lanes of the Illumina flow cell as technical replicates. Sequencing data from our study were submitted to National Center for Biotechnology Information (NCBI) under the accession number SRP080791. The GBS sequences were analyzed using the bioinformatics pipeline UNEAK [24], after which the tag sequence was converted into genotypic calls. Polymorphic parental GBS-SNPs were classified into eight segregation patterns, $\operatorname{lm} \times 1 \mathrm{ll}, \mathrm{ab} \times \mathrm{cd}, \mathrm{hk} \times \mathrm{hk}, \mathrm{ab} \times \mathrm{cc}, \mathrm{ef} \times \mathrm{eg}, \mathrm{cc} \times \mathrm{ab}$, aa $\times$ bb and $n n \times n p$; but aa and bb were considered for the $F_{2}$ population. To ensure linkage map quality, the GBS-SNPs markers used for the final mapping were selected by removing markers with significant distortion (Chi-square 1:2:1 test $p<0.001$ ) and more than $25 \%$ missing data.

\section{Genetic map construction and mapping of Pis1}

JoinMap Version 4.0 was utilized to construct the linkage map with GBS-SNP data [25]. Map construction excluded markers with obvious segregation distortion from the expected Mendelian segregation ratios of 1:2:1. Markers were positioned on linkage groups based on independence LOD threshold values of 2.0-12.0. We selected the JoinMap 'similarity of loci' command to identify identical markers (similarity value $=1.000$ ), which should be mapped to the same position on the linkage group. To reduce the calculation burden, only one marker of 'similar loci' was retained on the linkage map. Linkage analysis and marker order assignment were carried out using the regression mapping algorithm. Recombination fractions between markers were converted to map distances in $\mathrm{cM}$ using the Kosambi mapping function [26]. The final map included bin markers (excluding similar SNPs markers). The linkage maps were drawn using MapChart 2.2 [27]. All bin markers were compared with the bread wheat genome sequence, IWGSC1+popseq.31.pep (ftp://ftp. ensemblgenomes.org/pub/plants/release-31/fasta/triticum_ aestivum/pep/), by BLASTn.

To map Pis1, we assumed that the Pis1 locus is a quantitative trait locus (QTL) site and then used QTL analysis to locate Pis1. QTLs for the three-pistil phenotype were identified using the Interval Mapping program by MapQTL 6.0 [28]. The LOD threshold for significance for the presence of a putative QTL was 2.5. QTL peak positions were estimated using maximum LOD values. The phenotypic and SNP markers for three pistils on the 2D chromosome were then combined to map Pis1.

\section{Kompetitive allele-specific PCR (KASP) assay and candi- date gene identification}

KASP genotyping assays were developed for GBS-SNPs surrounding the Pis1 gene. Two allele-specific forward primers and one common reverse primer were designed for each KASP assay (Table 2). The KASP assays were carried out in 96-well PCR plates in a $5-\mu \mathrm{L}$ final volume consisting of $2.5 \mu \mathrm{L}$ KASP $2 \times$ Reaction Mix, $0.07 \mu \mathrm{L}$ assay mix $[12 \mu \mathrm{L}$ each allele-specific forward primer $(100 \mu \mathrm{M}), 30 \mu \mathrm{L}$ reverse primer $(100 \mu \mathrm{M})$, and $46 \mu \mathrm{L}$ TRIS (10 mM, pH 8.3)], and $50 \mathrm{ng}$ genomic DNA. The PCR program was performed under the following conditions: $94{ }^{\circ} \mathrm{C}$ for $15 \mathrm{~min} ; 10$ touchdown cycles of $94{ }^{\circ} \mathrm{C}$ for $20 \mathrm{~s}, 65-57{ }^{\circ} \mathrm{C}$ for $60 \mathrm{~s}$ (decreasing by $0.8{ }^{\circ} \mathrm{C}$ per cycle); and 32 cycles of $94{ }^{\circ} \mathrm{C}$ for $20 \mathrm{~s}, 57{ }^{\circ} \mathrm{C}$ for $60 \mathrm{~s}$. Fluorescence detection of the PCR reactions was carried out with a Bio-Rad CFX96 real-time PCR platform (Bio-Rad Laboratories, Hercules, CA, USA). KlusterCaller software (LGC Genomics, Beverly, USA) was applied to analyze the data.

The sequences of the markers flanking the Pis1 intervals were aligned back to the bread wheat genome sequence IWGSC1+popseq.31.pep (ftp://ftp.ensemblgenomes.org/ pub/plants/release-31/fasta/triticum_aestivum/pep/). All genes within the interval were identified as candidate genes based on the position of the flanking markers. All candidate genes were categorized by GO analysis (http:// www.geneontology.org/).

\section{Additional file}

Additional file 1: Table S1. GBS makers and their BLAST hit information. Table S2 Genes located in the intervals of Pis 1. Figure S1 High-density genetic map marker information. (DOCX $1044 \mathrm{~kb}$ ) 


\section{Abbreviations}

CM28: Chuanmai 28; GBS: Genotyping-by-sequencing; KASP: Kompetitive allele-specific PCR; MSG: Multiplexed shotgun sequencing; NGS: Next-generation sequencing; RAD-seq: Restriction-site-associated DNA sequencing; SNP: Singlenucleotide polymorphism; SSR: Simple sequence repeat; TP: Wheat mutant line three pistil

\section{Acknowledgements}

The authors thank Nature Research Editing Service, a company dedicated to helping international researchers publish their findings in the best English language, for editing the language.

\section{Funding}

This work was financially supported by the National Natural Science Foundation of China (Grant No. 31301319), the Foundation of Science \& Technology Department of Sichuan Province, China (Grant No. 16JC0022) and the project of Youth Science and Technology Innovation Team of Sichuan Province, China (Grant No. 2017TD0008). In all cases the funding body played no role in the design of the study and collection, analysis, and interpretation of data and in writing the manuscript

\section{Availability of data and materials}

The sequencing data from our study was deposited in the National Center for Biotechnology Information (NCBI) under the accession number SRP080791.

\section{Authors' contributions}

$Z Y$ designed the experiments and drafted the manuscript. $Z Y$ and $Z C$ carried out the phenotyping and genotyping of the F2 progeny. ZP participated in the language editing. YY constructed the F2 population. ML and SW participated in the data analysis. All authors read and approved the final manuscript.

\section{Ethics approval and consent to participate}

All the materials in this studies are cultivated by the authors, so there were no specific permits were required. Vouchers of those species have been placed in the China West Normal University Herbarium (CWNU).

\section{Consent for publication}

N/A.

\section{Competing interests}

The authors declare that they have no competing interests.

\section{Publisher's Note}

Springer Nature remains neutral with regard to jurisdictional claims in published maps and institutional affiliations.

Received: 18 December 2016 Accepted: 25 July 2017 Published online: 31 July 2017

\section{References}

1. Dencic S. Designing a wheat ideotype with increased sink capacity. Plant Breed. 1994;112:311-7.

2. Frederic JR, Bauer PJ. Physiological and numerical components of wheat yield. In: Satorre EH, Slafer GA, editors. Wheat ecology and physiologyof yield determination. New York: Food Products Press; 2000. p. 45-65.

3. Peng ZS, Yen C, Yang JL. Chromosomal location of genes for supernumerary spikelet in bread wheat. Euphytica. 1998:103:109-14.

4. Martinek P, Bednar J. Changes of spike morphology (multirowspike-MRS, long glumes-LG) in wheat (Triticum aestivum L.) and their importance for breeding, In: The proceedings of international conference 'genetic collections, isogenic and alloplasmiclines' Novosibirsk, Russia, 2001; 192-4.

5. Peng ZS. A new mutation in wheat producing three pistils in a floret. J Agron Crop Sci. 2003;189:270-2.

6. Peng ZS, Yang J, Wei SH, Zeng JH. Characterization of common wheat (Triticum aestivum L.) mutation line producing three pistils in a floret. Hereditas. 2004;141:15-8.

7. Peng ZS, Martinek P, Kosuge K, Kuboyama T. Genetic mapping of a mutan gene producing three pistils per floret in common wheat. J Appl Genet. 2008:49:135-9.
8. $\quad$ Yang ZJ, Peng ZS, Wei SH, Yu Y. Identification of differentially expressed genes in three-pistil mutation in wheat using annealing control primer system. Gene. 2011;485:81-4.

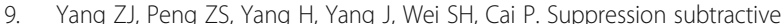
hybridization identified differentially expressed genes in pistil mutations in wheat. Plant Mol Bio Rep. 2011;29:431-9.

10. Yang ZJ, Peng ZS, Wei SH, Yan Y. Cloning and characterization of endobeta-1,4-glucanase genes in the common wheat line three pistils. Genet Mol Biol. 2013:36:400-7.

11. Yang ZJ, Peng ZS, Wei SH, Liao ML, Yu Y, Jang ZY. Pistilldoy mutant reveals key insights into stamen and pistil development in wheat (Triticum aestivum L.). BMC Genomics. 2015;16:211-20

12. Poland JA, Rife TW. Genotyping-by-sequencing for plant breeding and genetics. Plant Genome. 2012;5:92-102.

13. Poland JA, Brown PJ, Sorrells ME, Jannink JL. Development of high-density genetic maps for barley and wheat using a novel two-enzyme genotyping by-sequencing approach. PLoS One. 2012:7:251-64.

14. Somers DJ, Isaac P, Edwards K. A high-density microsatellite consensus map for bread wheat (Triticum aestivum L.). Theor Appl Genet. 2004:109:1105-14.

15. He J, Zhao X, Laroche A, Lu Z, Liu H, Li Z. Genotyping by sequencing (GBS), an ultimate marker-assisted selection (MAS) tool to accelerate plant breeding. Front Plant Sci. 2014:5:484-91.

16. Li G, Wang Y, Chen MS, Edae E, Poland J, Akhunov E, Chao S, Bai G, Carver B, Yan L. Precisely mapping a major gene conferring resistance to hessian fly in bread wheat using genotyping-by-sequencing. BMC Genomics. 2015;16:108-17.

17. Li H, Vikram P, Singh RP, Kilian A, Carling J, Song J, Burgueno-Ferreira JA Bhavani S, Huerta-Espino J, Payne T, Sehgal D, Wenzl P, Singh S. A high density GBS map of bread wheat and its application for dissecting complex disease resistance traits. BMC Genomics. 2015;16:216-30.

18. Cavanagh CR, Chao SM, Wang SC, Huang BE, Stephen S, Kiani S. Genomewide comparative diversity uncovers multiple targets of selection for improvement in hexaploid wheat landraces and cultivars. Proc Natl Acad Sci U S A. 2013;110:8057-62.

19. Wang SC, Wong D, Forrest K, Allen A, Chao S, Huang BE. Characterization of polyploid wheat genomic diversity using a high-density 90000 single nucleotide polymorphism array. Plant Biotechnol J. 2014;12:787-96.

20. Spindel J, Wright M, Chen C, Joshua Cobb J, Gage J, Harrington S, Lorieux M, Ahmadi N, McCouch S. Bridging the genotyping gap: using genotyping by sequencing (GBS) to add high-density SNP markers and new value to traditional bi-parental mapping and breeding populations. Theor Appl Genet. 2013;126:2699-716

21. Sonah H, Bastien M, lquira E, Tardivel A, Légaré G, Boyle B. An improved genotyping by sequencing (gbs) approach offering increased versatility and efficiency of snp discovery and genotyping. PLoS One. 2013:8:570.

22. Liu H, Bayer M, Druka A, Russell JR, Hackett CA, Poland J. An evaluation of genotyping by sequencing (GBS) to map the breviaristatum-e (ari-e) locus in cultivated barley. BMC Genomics. 2014;15:1-11.

23. Elshire RJ, Glaubitz JC, Sun Q, Poland JA, Kawamoto K, Buckler ES, Mitchell SE A robust, simple genotyping-by-sequencing (GBS) approach for high diversity species. PLoS One. 2011:6:1-10.

24. Lu F, Lipka AE, Glaubitz J, Elshire R, Cherney JH, Casler MD. Switchgrass genomic diversity, ploidy, and evolution: novel insights from a networkbased SNP discovery protocol. PLoS Genet. 2013;9:139-47.

25. van Ooijen JW. JoinMap ${ }^{\oplus} 4$, software for the calculation of genetic linkage maps in experimental populations. Wageningen, Netherlands: Kyazma BV; 2006

26. Kosambi D. The estimation of map distances from recombination values. Ann Eugenics. 1994;12:172-5.

27. Voorrips R. MapChart: software for the graphical presentation of linkage maps and QTLs. J Hered. 2002;93:77-8.

28. Van Oojjen JW. MapQTL 6, software for the mapping of quantitative trait loci in experimental populations of diploid species. Wageningen, Netherlands: Kyazma BW; 2009 